\title{
ВЫЧИСЛИТЕЛЬНАЯ ТЕХНИКА
}

\section{СИНТЕЗ КОМПЛЕКСНЫХ ДИСКРЕТНЫХ ФИЛЬТРОВ МЕТОДОМ ИНВАРИАНТНЫХ ДИФФЕРЕНЦИАЛЬНЫХ УРАВНЕНИЙ}

\author{
С. И. ЗИАТДИНОВ, Л. А. ОСИПОВ, Ю. В. СОКОЛОВА \\ Санкт-Петербургский государственный университет аэрокосмического приборостроения, \\ 190000, Санкт-Петербург, Россия \\ E-mail: kaf53@guap.ru
}

\begin{abstract}
Рассмотрена методика синтеза комплексных дискретных режекторных и селективных фильтров на основе дифференциальных уравнений непрерывных фильтров-аналогов с использованием разностных уравнений. Для получения разностных уравнений, описывающих работу синтезируемых комплексных дискретных фильтров, производные комплексных дифференциальных уравнений заменяются аналогами в виде комплексных разностных уравнений. При правильном выборе периода дискретности частотные свойства синтезированных фильтров практически совпадают с частотными свойствами соответствующих непрерывных фильтров. Приведены примеры проектирования комплексных дискретных режекторных и селективных фильтров методом инвариантных дифференциальных уравнений.
\end{abstract}

Ключевые слова: комплексный дискретный фильтр, дифференциальное уравнение, разностное уравнение, частотная передаточная функиия

В настоящее время в связи с интенсивным развитием вычислительной техники и появлением высокоэффективных цифровых устройств, таких как микропроцессоры, микроконтроллеры, сигнальные процессоры, наибольшее распространение получили цифровые методы обработки видеосигналов. Для повышения качества обработки сигналов, подавления помех и шумов широко используются разнообразные действительные и комплексные фильтры нижних и верхних частот, полосовые и режекторные фильтры. Применение комплексных фильтров позволяет сравнительно легко адаптировать их параметры к параметрам обрабатываемых сигналов. При этом в каждом конкретном случае к временным и частотным свойствам фильтров в зависимости от решаемых задач предъявляются специфические требования.

Вопросы построения действительных и комплексных дискретных фильтров с заданными временными и частотными характеристиками достаточно хорошо исследованы [1-3]. Для синтеза дискретных фильтров по их непрерывным аналогам используются как частотные, так и временные методы [4]. В основе частотных методов лежит применение билинейного $z$-преобразования, позволяющего по заданной частотной передаточной функции непрерывного фильтра разработать дискретный фильтр, частотные свойства которого практически совпадают с частотными свойствами непрерывного фильтра-аналога.

В случае синтеза комплексных дискретных фильтров во временной области используется либо метод инвариантной импульсной характеристики, либо метод инвариантной переходной характеристики $[5,6]$. Оба метода основаны на применении для создания комплексных дискретных фильтров отсчетов импульсной или переходной характеристик непрерывного 
фильтра-аналога. Вместе с тем возможен метод синтеза как действительных, так комплексных дискретных фильтров на основе дифференциальных уравнений, описывающих работу непрерывных фильтров. В литературе этому методу практически не уделено должного внимания. Однако использование дифференциальных уравнений позволяет сравнительно легко получать алгоритмы работы фильтров нижних и верхних частот, полосовых и режекторных фильтров.

Цель настоящей работы заключается в создании методики синтеза комплексных дискретных фильтров с использованием дифференциальных уравнений непрерывных комплексных фильтров-аналогов.

В самом общем виде комплексная линейная система (комплексный фильтр) порядка $n$ описывается следующим комплексным линейным дифференциальным уравнением [7]:

$$
\begin{gathered}
a_{0} \frac{d^{n} z_{\mathrm{Bdx}}^{*}(t)}{d t^{n}}+a_{1} \frac{d^{n-1} z_{\mathrm{Bdx}}^{*}(t)}{d t^{n-1}}+\ldots+a_{n} z_{\mathrm{Bdx}}^{*}(t)= \\
=b_{0} \frac{d^{m} z_{\mathrm{BX}}^{*}(t)}{d t^{m}}+b_{1} \frac{d^{m-1} z_{\mathrm{BX}}^{*}(t)}{d t^{m-1}}+\ldots+b_{m} z_{\mathrm{BX}}^{*}(t), m \leq n,
\end{gathered}
$$

где $z_{\text {вых }}^{*}(t)=z_{\text {вых }}(t) \exp \left(j \omega_{0} t\right) \quad$ - комплексный выходной сигнал фильтра, $z_{\mathrm{BX}}^{*}(t)=u_{\mathrm{BX}}^{*}(t) \exp \left(-j \omega_{0} t\right)-$ комплексный входной сигнал $u_{\mathrm{BX}}^{*}(t)$, перенесенный на частоту настройки фильтра $\omega_{0} ; z_{\text {вых }}(t)-$ модуль комплексного выходного сигнала; $a_{i}, b_{i}-$ весовые коэффициенты.

Производные в соотношении (1) можно представить в виде

$$
\begin{aligned}
& \frac{d z_{\text {Bых }}^{*}(t)}{d t}=\lim _{T \rightarrow 0} \frac{z_{\text {вых }}^{*}(t)-z_{\text {вых }}^{*}(t-T)}{T} ; \\
& \frac{d^{2} z_{\mathrm{Bbx}}^{*}(t)}{d t^{2}}=\lim _{T \rightarrow 0} \frac{\frac{d z_{\mathrm{Bbx}}^{*}(t)}{d t}-\frac{d z_{\mathrm{Bbx}}^{*}(t-T)}{d t}}{T} ; \\
& \frac{d^{n} z_{\text {вых }}^{*}(t)}{d t^{n}}=\lim _{T \rightarrow 0} \frac{\frac{d^{n-1} z_{\text {Bых }}^{*}(t)}{d t}-\frac{d^{n-1} z_{\text {вых }}^{*}(t-T)}{d t}}{T} ; \\
& \frac{d z_{\mathrm{BX}}^{*}(t)}{d t}=\lim _{T \rightarrow 0} \frac{z_{\mathrm{BX}}^{*}(t)-z_{\mathrm{BX}}^{*}(t-T)}{T} ; \\
& \frac{d^{2} z_{\mathrm{BX}}^{*}(t)}{d t^{2}}=\lim _{T \rightarrow 0} \frac{\frac{d z_{\mathrm{BX}}^{*}(t)}{d t}-\frac{d z_{\mathrm{BX}}^{*}(t-T)}{d t}}{T} ; \\
& \frac{d^{m} z_{\mathrm{BX}}^{*}(t)}{d t^{m}}=\lim _{T \rightarrow 0} \frac{\frac{d^{m-1} z_{\mathrm{BX}}^{*}(t)}{d t^{m-1}}-\frac{d^{m-1} z_{\mathrm{BX}}^{*}(t-T)}{d t^{m-1}}}{T} .
\end{aligned}
$$

В дальнейшем будем считать, что за время $T$ не происходит заметных изменений ни функций $z_{\text {вх }}^{*}(t), z_{\text {вых }}^{*}(t)$, ни их производных.

Тогда соотношения (2) можно заменить приближенными разностными уравнениями

$$
\frac{d z_{\mathrm{Bbx}}^{*}(t)}{d t} \approx \frac{z_{\mathrm{Bbx}}^{*}(t)-z_{\text {вых }}^{*}(t-T)}{T} ;
$$




$$
\begin{gathered}
\frac{d^{2} z_{\mathrm{BbX}}^{*}(t)}{d t^{2}} \approx \frac{\frac{d z_{\mathrm{Bdx}}^{*}(t)}{d t}-\frac{d z_{\mathrm{BdX}}^{*}(t-T)}{d t}}{T} ; \\
\frac{d^{n} z_{\mathrm{Bbx}}^{*}(t)}{d t^{n}} \approx \frac{\frac{d^{n-1} z_{\mathrm{Bbx}}^{*}(t)}{d t}-\frac{d^{n-1} z_{\mathrm{Bdx}}^{*}(t-T)}{d t}}{T} ; \\
\frac{d z_{\mathrm{BX}}^{*}(t)}{d t} \approx \frac{z_{\mathrm{BX}}^{*}(t)-z_{\mathrm{BX}}^{*}(t-T)}{T} ; \\
\frac{d^{2} z_{\mathrm{BX}}^{*}(t)}{d t^{2}} \approx \frac{\frac{d z_{\mathrm{BX}}^{*}(t)}{d t}-\frac{d z_{\mathrm{BX}}^{*}(t-T)}{d t}}{T} ; \\
\frac{d^{m} z_{\mathrm{BX}}^{*}(t)}{d t^{m}} \approx \frac{\frac{d^{m-1} z_{\mathrm{BX}}^{*}(t)}{d t^{m-1}}-\frac{d^{m-1} z_{\mathrm{BX}}^{*}(t-T)}{d t^{m-1}}}{T} .
\end{gathered}
$$

В таблице представлены значения весовых коэффициентов $c_{i}$ разностных уравнений, соответствующих производным различных порядков.

Весовые коэффициенты

\begin{tabular}{|c|c|c|c|c|c|c|c|c|c|c|}
\hline$n$ & $c_{0}$ & $c_{1}$ & $c_{2}$ & $c_{3}$ & $c_{4}$ & $c_{5}$ & $c_{6}$ & $c_{7}$ & $c_{8}$ & $c_{9}$ \\
\hline 1 & 1 & -1 & & & & & & & & \\
\hline 2 & 1 & -2 & 1 & & & & & & & \\
\hline 3 & 1 & -3 & 3 & -1 & & & & & & \\
\hline 4 & 1 & -4 & 6 & -4 & 1 & & & & & \\
\hline 5 & 1 & -5 & 10 & -10 & 5 & -1 & & & & \\
\hline 6 & 1 & -6 & 15 & -20 & 15 & -6 & 1 & & & \\
\hline 7 & 1 & -7 & 21 & -35 & 35 & -21 & 7 & -1 & & \\
\hline 8 & 1 & -8 & 28 & -56 & 70 & -56 & 28 & -8 & 1 & \\
\hline 9 & 1 & -9 & 36 & -84 & 126 & -126 & 84 & -36 & 9 & -1 \\
\hline
\end{tabular}

С учетом соотношений (3) дифференциальное уравнение линейной системы (1) записывается в виде конечных разностей

$$
\begin{aligned}
& a_{0} T^{-n} \sum_{i=0}^{n} c_{i} z_{\text {вых }}^{*}(t-i T)+a_{1} T^{-(n-1)} \sum_{i=0}^{n-1} p_{i} z_{\mathrm{Bbx}}^{*}(t-i T)+\ldots+a_{n} z_{\mathrm{Bыx}}^{*}(t)= \\
& =b_{0} T^{-m} \sum_{i=0}^{m} k_{i} z_{\mathrm{BX}}^{*}(t-i T)+b_{1} T^{-(m-1)} \sum_{i=0}^{m-1} d_{i} z_{\mathrm{BX}}^{*}(t-i T)+\ldots+b_{m} z_{\mathrm{BX}}^{*}(t) .
\end{aligned}
$$

В выражении (4) весовые коэффициенты $c_{i}$ соответствуют $n$-й производной; $p_{i}-(n-1)$-й производной; $k_{i}-m$-й производной; $d_{i}-(m-1)$-й производной и т.д.

Из соотношения (4) находим выходной сигнал фильтра

$$
\begin{gathered}
z_{\text {вых }}^{*}(t)=a_{n}^{-1}\left[b_{0} T^{-m} \sum_{i=0}^{m} k_{i} z_{\mathrm{Bx}}^{*}(t-i T)+b_{1} T^{-(m-1)} \sum_{i=0}^{m-1} d_{i} z_{\mathrm{Bx}}^{*}(t-i T)+\ldots+b_{m} z_{\mathrm{Bx}}^{*}(t)-\right. \\
\left.-a_{0} T^{-n} \sum_{i=0}^{n} c_{i} z_{\text {вых }}^{*}(t-i T)+a_{1} T^{-(n-1)} \sum_{i=0}^{n-1} p_{i} z_{\mathrm{Bыx}}^{*}(t-i T)+\ldots+a_{n} z_{\text {вых }}^{*}(t)\right] .
\end{gathered}
$$

При дискретном представлении входного и выходного сигналов фильтра, когда $t=i T$, работа дискретного фильтра на основании (5) определяется выражением 


$$
\begin{gathered}
z_{\text {вых }}^{*}[n]=a_{n}^{-1}\left\{b_{0} T^{-m} \sum_{i=0}^{m} k_{i} z_{\text {вх }}^{*}[n-i]+b_{1} T^{-(m-1)} \sum_{i=0}^{m-1} d_{i} z_{\text {вх }}^{*}[n-i]+\ldots+b_{m} z_{\text {вх }}^{*}[n]-\right. \\
\left.\quad-a_{0} T^{-n} \sum_{i=0}^{n} c_{i} z_{\text {вых }}^{*}[n-i]+a_{1} T^{-(n-1)} \sum_{i=0}^{n-1} p_{i} z_{\text {вых }}^{*}[n-i]+\ldots+a_{n} z_{\text {вых }}^{*}[n]\right\} .
\end{gathered}
$$

Это соотношение определяет алгоритм работы дискретного фильтра порядка $n$. Рассмотрим ряд конкретных примеров.

Комплексный селективный фильтр третьего порядка. Частотная передаточная функция непрерывного фильтра и его дифференциальное уравнение имеют вид [7]:

$$
\begin{gathered}
W\left[j\left(\omega-\omega_{0}\right)=1 /\left\{1+\left[j\left(\omega-\omega_{0}\right) \tau\right]^{3}\right\} ;\right. \\
\tau^{3} \frac{d^{3} z_{\text {вых }}^{*}(t)}{d t^{3}}+z_{\text {вых }}^{*}(t)=z_{\text {вх }}^{*}(t),
\end{gathered}
$$

где $\tau$ - постоянная времени фильтра.

После замены в дифференциальном уравнении третьей производной ее разностным эквивалентом (третья строчка в таблице) получим следующее разностное уравнение селективного фильтра третьего порядка:

где $a=(\tau / T)^{3}$.

$$
z_{\text {вых }}^{*}[n]=\frac{1}{1+a} z_{\text {вх }}^{*}[n]+\frac{a}{1+a}\left\{3 z_{\text {вых }}^{*}[n-1]-3 z_{\text {вых }}^{*}[n-2]+z_{\text {вых }}^{*}[n-2]\right\},
$$

Выражение (7) позволяет записать амплитудно-частотную характеристику (АЧХ) рассматриваемого фильтра в виде

$$
\begin{aligned}
W(\omega)= & \frac{1}{1+a} /\left\{1-\frac{a}{1+a}\left\{\left[3 \cos \left[\left(\omega-\omega_{0}\right) T\right]-3 \cos \left[2\left(\omega-\omega_{0}\right) T\right]+\cos 3\left[\left(\omega-\omega_{0}\right) T\right]\right\}^{2}+\right.\right. \\
& \left.+\frac{a}{1+a}\left\{3 \sin \left[\left(\omega-\omega_{0}\right) T\right]-3 \sin \left[2\left(\omega-\omega_{0}\right) T\right]+\sin \left[3\left(\omega-\omega_{0}\right) T\right]\right\}^{2}\right\}^{0,5} .
\end{aligned}
$$

Результаты расчета АЧХ рассматриваемого комплексного селективного дискретного фильтра для случая $a=(\tau / T)^{3}=10^{3}, f_{0}=\omega_{0} / 2 \pi=-10$ Гц приведены на рисунке (кривая 1$)$. Отметим, что при частоте настройки фильтра $f_{0}=0$ селективный фильтр становится фильтром нижних частот.

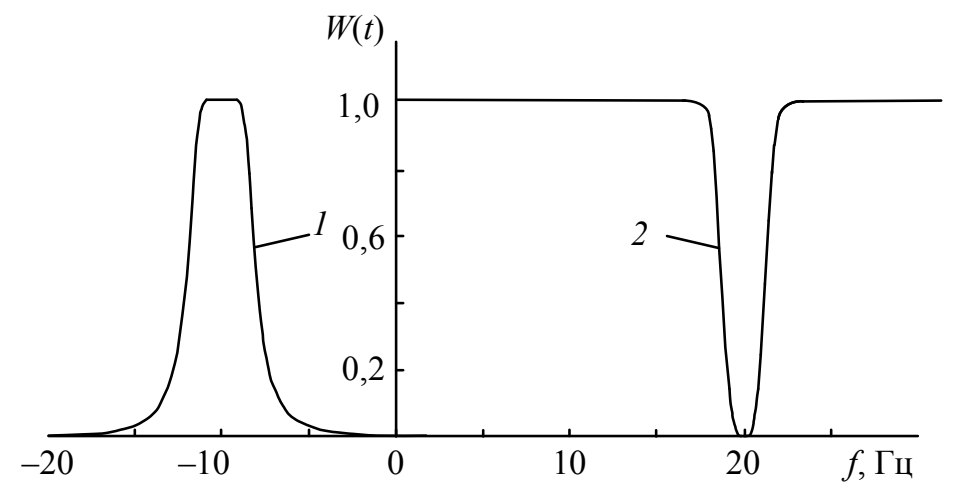

Режекторный фильтр третьего порядка. В этом случае частотная передаточная функция и дифференциальное уравнение описываются соотношениями [7]:

$$
\begin{gathered}
W\left[j\left(\omega-\omega_{0}\right)=\left[j\left(\omega-\omega_{0}\right) \tau\right]^{3} /\left\{1+\left[j\left(\omega-\omega_{0}\right) \tau\right]^{3}\right\} ;\right. \\
\tau^{3} \frac{d^{3} z_{\text {вых }}^{*}(t)}{d t^{3}}+z_{\text {вых }}^{*}(t)=\frac{d^{3} z_{\text {вх }}^{*}(t)}{d t^{3}} .
\end{gathered}
$$


Применив представленную ранее методику, получим для комплексного дискретного режекторного фильтра разностное уравнение вида

$$
\begin{aligned}
z_{\text {вых }}^{*}[n]= & \frac{a}{1+a}\left\{z_{\mathrm{BX}}^{*}[n]-3 z_{\mathrm{BX}}^{*}[n-1]+3 z_{\mathrm{BX}}^{*}[n-2]-z_{\mathrm{BX}}^{*}[n-3]-\right. \\
& \left.-3 z_{\text {вых }}^{*}[n-1]+3 z_{\text {вых }}^{*}[n-2]-z_{\text {вых }}^{*}[n-3]\right\} .
\end{aligned}
$$

Тогда АЧХ рассматриваемого комплексного фильтра запишется следующим образом:

$$
W(\omega)=\frac{\frac{a}{1+a} \sqrt{c_{1}+c_{2}}}{\sqrt{b_{1}+b_{2}}},
$$

где

$$
\begin{gathered}
c_{1}=\left\{1-3 \cos \left[\left(\omega-\omega_{0}\right) T\right]+3 \cos \left[2\left(\omega-\omega_{0}\right) T\right]-\cos \left[3\left(\omega-\omega_{0}\right) T\right]\right\}^{2} ; \\
c_{2}=\left\{3 \sin \left[\left(\omega-\omega_{0}\right) T\right]-3 \sin \left[2\left(\omega-\omega_{0}\right) T\right]+\sin \left[3\left(\omega-\omega_{0}\right) T\right]\right\}^{2} ; \\
b_{1}=\left\{1-\frac{a}{1+a}\left\{3 \cos \left[\left(\omega-\omega_{0}\right) T\right]-3 \cos \left[2\left(\omega-\omega_{0}\right) T\right]+\cos \left[3\left(\omega-\omega_{0}\right) T\right]\right\}\right\}^{2} ; \\
b_{2}=\left\{1-\frac{a}{1+a}\left\{3 \cos \left[\left(\omega-\omega_{0}\right) T\right]-3 \cos \left[2\left(\omega-\omega_{0}\right) T\right]+\cos \left[3\left(\omega-\omega_{0}\right) T\right]\right\}\right\}^{2} .
\end{gathered}
$$

На рисунке для $a=(\tau / T)^{3}=10^{3}, f_{0}=\omega_{0} / 2 \pi=20$ Гц приведена АЧХ комплексного дискретного режекторного фильтра (кривая 2). При частоте настройки фильтра $f_{0}=0$ режекторный фильтр становится фильтром верхних частот.

Рассмотренная методика позволяет синтезировать комплексные дискретные фильтры нижних и верхних частот, селективные и режекторные фильтры на основе известных комплексных дифференциальных уравнений непрерывных фильтров-аналогов с использованием разностных уравнений. Частотные свойства синтезированных комплексных дискретных фильтров при правильном выборе периода дискретности практически совпадают с частотными свойствами соответствующих непрерывных фильтров. Комплексные фильтры позволяют легко изменять их частоту настройки. При этом форма АЧХ фильтров остается прежней.

\section{СПИСОК ЛИТЕРАТУРЫ}

1. Воробьев С. Н. Цифровая обработка сигналов. М.: Академия, 2013.318 с.

2. Голд Б., Рейдер Ч. Цифровая обработка сигналов. М.: Сов. радио, 1973. 367 с.

3. Сергиенко А. Б. Цифровая обработка сигналов. СПб: БВХ-Петербург, 2015. 756 с.

4. Оппенгейм А., Шафер Р. Цифровая обработка сигналов. М.: Техносфера, 2006. 855 с.

5. Зиатдинов С. И. Анализ линейных систем на основе переходных характеристик // Информационноуправляющие системы. 2016. № 2. С. 104-106.

6. Зиатдинов С. И. Синтез рекурсивных дискретных фильтров во временной области // Изв. вузов. Радиоэлектроника. 2016. № 3. С. 3-6.

7. Гоноровский И. С. Радиотехнические цепи и сигналы. М.: Радио и связь, 1986. 512 с.

\section{Сведения об авторах}

Сергей Ильич Зиатдинов

Леонид Андроникович Осипов
- д-р техн. наук, профессор; СПбГУАП, кафедра информационносетевых технологий; E-mail: kaf53@guap.ru

- д-р техн. наук, профессор; СПбГУАП, кафедра информационносетевых технологий; E-mail: kaf53@guap.ru 
Ссылка для цитирования: Зиатдинов С. И., Осипов Л. А., Соколова Ю. В. Синтез комплексных дискретных фильтров методом инвариантных дифференциальных уравнений // Изв. вузов. Приборостроение. 2018. Т. 61, № 4. С. $317-322$.

\section{SYNTHESIS OF COMPLEX DISCRETE FILTERS BY METHOD OF INVARIANT DIFFERENTIAL EQUATIONS}

\section{S. I. Ziatdinov, L. A. Osipov, Yu. V. Sokolova}

St. Petersburg State University of Aerospace Instrumentation, St. Petersburg, 190000, St. Petersburg, Russia

E-mail:kaf53@guap.ru

A technique for synthesis of various complex discrete linear systems on the base of differential equations for continuous analog filters is considered. The systems include low and high frequency filters, vibrational links, rejection and selective filters. To derive the difference equations describing the operation of synthesized linear systems, the derivatives of differential equations are replaced by their analogs in the form of complex difference equations. With the correct choice of discretization period, the frequency properties of synthesized discrete filters coincide practically with the frequency properties of the respective continuous filters. Specific examples of design of complex discrete filters of low and high frequencies using the method of invariant selective differential equations are presented. function

Keywords: complex discrete filter, differential equation, separation equation, frequency transfer

\section{Data on authors}

Sergey I. Ziatdinov

- Dr. Sci., Professor; St. Petersburg State University of Aerospace Instrumentation, Department of Information and Network Technologies; E-mail: kaf53@guap.ru

Leonid A. Osipov — Dr. Sci., Professor; St. Petersburg State University of Aerospace Instrumentation, Department of Information and Network Technologies; E-mail: kaf53@guap.ru

Yulia V. Sokolova

- Post-Graduate Student; St. Petersburg State University of Aerospace Instrumentation, Department of Information and Network Technologies; E-mail: kaf53@guap.ru

For citation: Ziatdinov S. I., Osipov L. A., Sokolova Yu. V. Synthesis of complex discrete filters by method of invariant differential equations. Journal of Instrument Engineering. 2018. Vol. 61, N 4. P. 317-322 (in Russian).

DOI: 10.17586/0021-3454-2018-61-4-317-322 